\title{
Evaluation and Design Exploration of Solar Harvested-Energy Prediction Algorithm
}

\author{
Mustafa Imran Ali, Bashir M. Al-Hashimi \\ School of Electronics and Computer Science \\ University of Southampton \\ Southampton, UK \\ Email: \{mia08r, bmah\}@ecs.soton.ac.uk
}

\author{
Joaquín Recas \\ DACYA \\ Complutense University \\ Madrid, Spain \\ Email: jrecas@fis.ucm.es
}

\author{
David Atienza \\ Embedded Systems Laboratory \\ Ecole Polytechnique Fédérale de Lausanne \\ Lausanne, Switzerland \\ Email: david.atienza@epfl.ch
}

\begin{abstract}
To respond to variations in solar energy, harvestedenergy prediction is essential to harvested-energy management approaches. The effectiveness of such approaches is dependent on both the achievable accuracy and computation overhead of prediction algorithm implementation. This paper presents detailed evaluation of a recently reported solar energy prediction algorithm to determine empirical bounds on achievable accuracy and implementation overhead using an effective error evaluation technique. We evaluate the algorithm performance over varying prediction horizons and propose guidelines for algorithm parameter selection across different real solar energy profiles to simplify implementation. The prediction algorithm computation overhead is measured on actual hardware to demonstrate prediction accuracy-cost trade-off. Finally, we motivate the basis for dynamic prediction algorithm and show that more than $10 \%$ increase in prediction accuracy can be achieved compared to static algorithm.
\end{abstract}

\section{INTRODUCTION}

Use of ambient energy harvesting to design perpetually powered embedded systems (Fig. 1) has received much attention in recent years [1]. Realizing such systems that meet application's performance and availability requirements may be non-trivial depending upon many factors. This includes efficiency of energy harvester in converting ambient energy to electrical energy, non-ideal behaviour of power extraction circuits and energy storage, constraints on sizes of harvester and energy storage capacity, and variability of ambient energy source. Solar energy harvesting has been increasingly deployed to design energy harvesting embedded systems due to its relatively high harvested energy density and falling costs of

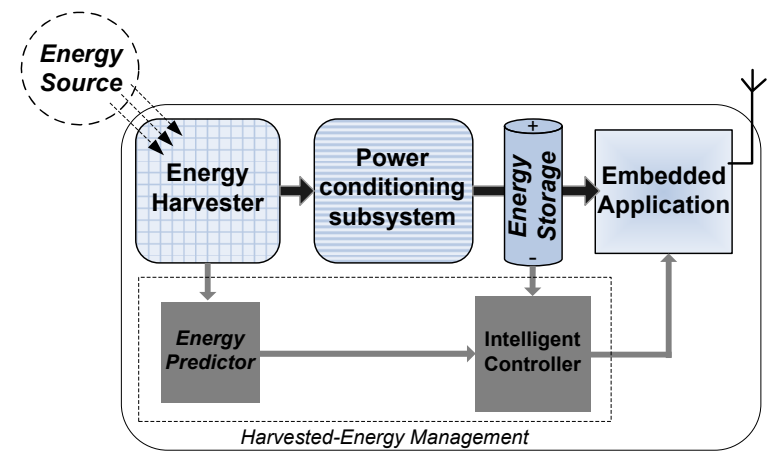

Fig. 1. Components of a typical energy harvesting embedded system with harvested-energy management functions

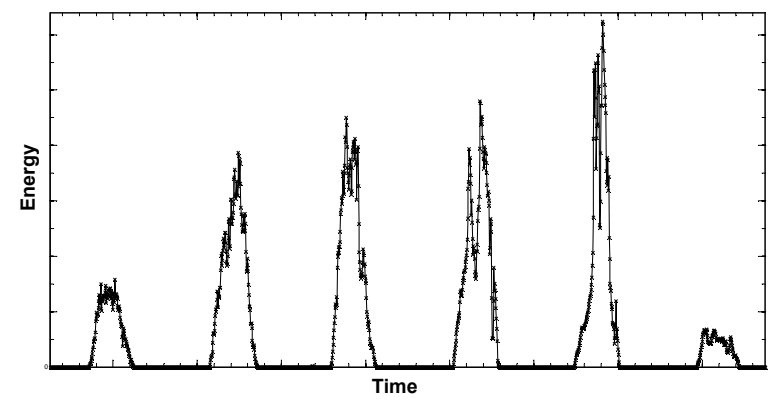

Fig. 2. Solar energy measured on 6 days [6] showing variation in energy received during different times in a day and across days. Each point represents energy received during a 5 minutes interval

photovoltaic panels [1]. The amount of solar energy received at different times in a day and across days can vary significantly (Fig. 2). Under these conditions, considering the constraints on system components, the performance requirements can be met by constantly adapting the application's energy consumption to match energy harvested from the environment. To this end, approaches for harvested-energy management have been proposed that adapt the system to uncertainty in energy availability by predicting the expected incoming energy $[2,3,4,5]$. In case of solar energy, this is possible by capitalizing on its 24-hour cycles to predict energy availability.

Kansal et al. [2] were the first to propose a simple solar energy prediction algorithm to support their harvested-energy management approach. The predictor was based on the observation that energy generation during a given time slot of day was similar to that generated at the same instant on previous days and it can be estimated using an exponentially weighted moving average of historical data. Moser et al. [3] used similar prediction in their proposed adaptive power management framework. Recently, Recas et al. [5] proposed an improved solar energy predictor by using past and current day's power measurements in the prediction algorithm. Noh et al. [4] have used a similar principle for prediction in their minimum variance energy allocation. Recently, Bergonzini et al. [7] compare the prediction error and computation requirements of different prediction algorithms.

The effectiveness of harvested-energy management $[2,3,4,5]$ is sensitive to accuracy of prediction algorithm. This has been acknowledged in previous works, nevertheless, there 
is a lack of clear justification how the prediction accuracy should be best quantified. The accuracy of prediction algorithm is dependent upon parameters such as number of samples of energy source taken per day or the length of prediction horizon and window sizes of historical source data samples used. At the same time, these parameters also determine the overhead of performing prediction algorithm operations and memory requirement for storing historical power samples. Since harvested power is often limited, it is important to minimize the energy consumption overhead of harvested-energy management activity, including prediction. In previous works $[2,3,4,5,7]$ the choice of prediction parameters have been based largely on specific cases, and no comprehensive evaluation has been presented using different parameter choices and across multiple data sets that clearly identifies the extent of trade-off between prediction accuracy and its cost.

In this paper we consider how prediction accuracy of solar harvested-energy can be evaluated and applied to evaluate achievable accuracy of a recently reported improved solar energy prediction algorithm [5] using multiple real solar energy data sets. The algorithm performance is measured by varying energy harvesting source sampling rates (or prediction horizons) and trade-off in prediction accuracy and cost is obtained based on implementing prediction algorithm on actual hardware. We analyze results across different working conditions to give guidelines to simplify prediction algorithm's parameters tuning, which ensures that high accuracies can be achieved without the need to optimize for different working conditions. Finally, we motivate the case for dynamic parameters prediction and show that on average greater than $10 \%$ higher accuracies can be achieved compared to the static algorithm. The paper is organized as follows. The prediction algorithm is explained in Section II. Section III discusses the prediction error measurement method. Section IV presents a detailed evaluation of prediction algorithm followed by a discussion of dynamic prediction. Section $\mathrm{V}$ concludes the paper.

\section{Prediction Algorithm}

To present detailed analysis on prediction accuracy and overhead, we first describe the prediction algorithm in [5] and introduce the algorithm's parameters. For energy management and prediction, a day is discretized into $N$ equal duration time slots. Incoming power sampling and prediction are performed once per slot and the slot's length is the prediction horizon. To predict the future slot power, the algorithm [5] uses measured power values $e(i, j) \in \mathbf{E}_{D \times N}$ of the last $D \in \mathbb{Z}^{+}$days' slots. It also uses measured power values $\widetilde{e}(j) \in \widetilde{\mathbf{E}}_{N}$ of the current day's elapsed slots. The matrix $\mathbf{E}_{D \times N}$ and the vector $\widetilde{\mathbf{E}}_{N}$ are shown in Fig. 3. Assume that at present $n \in N$ slots have elapsed on the current day shown shaded in Fig. 3 and $\widetilde{e}(n)$ is the measured power value at start of slot $n$. The power $\hat{e}_{n+1}$ at the beginning of slot $n+1$ (marked with a '?', Fig. 3) needs to be predicted. In Fig. 3, $\mu_{D}(n+1)$ denotes average of power measured at beginning of all $n+1$ slots in last $D$ days. The predicted power is a combination of present slot power $\widetilde{e}(n)$ and the average $\mu_{D}(n+1)$ of predicted slot $(n+1)$ :

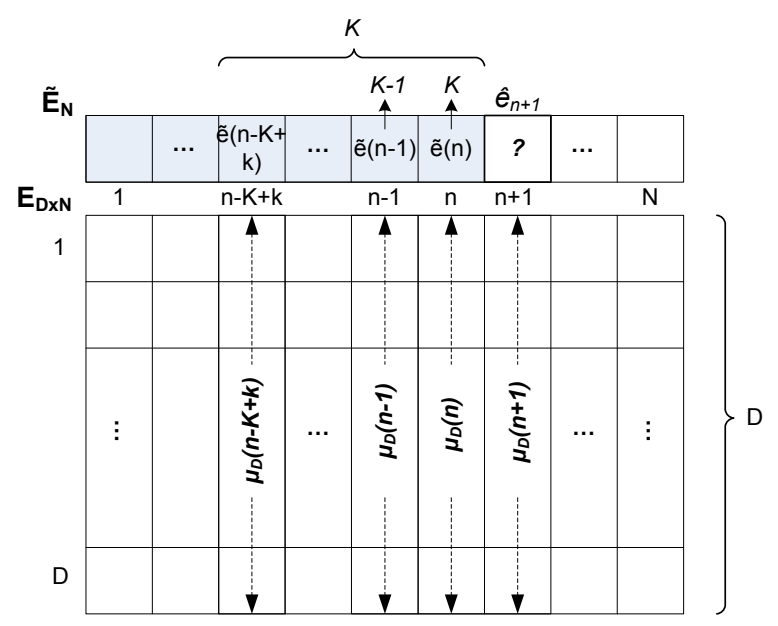

Fig. 3. Graphical depiction of the prediction algorithm [5]

$$
\hat{e}_{n+1}=\alpha \cdot \widetilde{e}(n)+(1-\alpha) \cdot \mu_{D}(n+1) \cdot \Phi_{K}
$$

In Equation 1, $\alpha$ is a weighting parameter with value $0 \leq$ $\alpha \leq 1$. The determination of $\alpha$ and other algorithm parameters is explained shortly. $\mu_{D}(j)$ is the average of power measured at beginning of slots $j \in N$ in the past $D$ days:

$$
\mu_{D}(j)=\frac{\sum_{i=1}^{D} e(i, j)}{D}
$$

$\Phi_{K}$ is a conditioning factor for $\mu_{D}(n+1)$ and its computation depends on parameter $K \in \mathbb{Z}^{+}$, which is the number of slots considered before slot $(n+1)$ of the current day (Fig. 3). $\Phi_{K}$ is a measure of how much brighter or cloudy the current day is compared to previous days [5]. It is evaluated using Equation 3, which is a weighted average of ratios $\eta(k) \in H_{K}$ (Equation 4), where each ratio $\eta(k)$ compares the current day's measured power (of a slot) to past days' average. The weights $\theta(k) \in \Theta_{K}$ (Equation 5) decrease from 1 to $\frac{1}{K}$ starting at slot $n$ since slots earlier than $n$ are assumed to be less correlated to the future slot $(n+1)$ [5].

$$
\begin{gathered}
\Phi_{K}=\frac{\left(\Theta_{K}\right)^{T} \cdot H_{K}}{\sum_{k=1}^{K} \theta(k)} \\
\eta(k)=\frac{\widetilde{e}(n-K+k)}{\mu_{D}(n-K+k)} \\
\theta(k)=\frac{k}{K}
\end{gathered}
$$

The predicted power given by the algorithm in [5] consists of two terms, Equation 1. In this paper, the first of these terms is labeled as the persistence term and the second one is the conditioned average term. The persistence term determines how much slot $n$ power value contributes to the predicted value, while the conditioned average term is the contribution of averaged past $(n+1)$ th slots scaled by the conditioning factor $\Phi_{K}$. The parameter $\alpha$ weighs these two contributions. Fig. 3 shows that the parameter $D$ controls how many past 
days influence the predicted value, while the parameter $K$ determines the influence of previous slots of the current day.

For a given sampling rate of solar power per day $(N)$, to select values of parameters $\alpha, D$, and $K$ from their set of possible values, Equation 1 is evaluated using different values of each parameter over a target solar power data set to find the minimum value of an error function. The value of error function depends on input solar power data and the parameters' $(\alpha, D, K$ and $N)$ selected values. The values of parameters $\alpha, D$, and $K$ that minimize the error function at a given sampling rate $(N)$ is the optimized set. The next section presents the details of the proposed error evaluation technique.

\section{iII. Prediction Error Measurement}

In this section we discuss an error evaluation technique that accurately models the prediction error and is intuitive to allow comparison of prediction algorithm across different solar power data sets. We suggest how predicted output should be compared so that the result is representative of the actual error and which error function to use to compute averaged error to model overall losses in prediction. To motivate our error calculation technique, Figure 4 shows a section of measured solar power profile of a day. Slot boundaries are indicated and in each slot $M$ power samples are available. For instance, if slot length is $T=30$ minutes $(N=48)$ and sampling resolution of available data is 5 minutes then $M=6$. Power samples at start of each slot are indicated and these are used by prediction algorithm (Section II) to predict future slot power. The energy received during a slot $n$ can be obtained from its mean power $\bar{e}_{n}$ by $\bar{e}_{n} \times T$. Harvested-energy management system estimates the energy of slot $n$ by using the predicted power value $\hat{e}_{n+1}$ as $\hat{e}_{n+1} \times T$.

In previous works [2,5], the prediction error of a slot $n$ $\left(\operatorname{error}_{n}^{\prime}\right)$ is expressed as:

$$
\operatorname{error}_{n}^{\prime}=e_{n+1}-\hat{e}_{n+1}
$$

Since predicted power is used to estimate a slot's energy, we argue that it is more realistic to compare the predicted power to mean power of a slot to express prediction error:

$$
\operatorname{error}_{n}=\bar{e}_{n}-\hat{e}_{n+1}
$$

The value of $\bar{e}_{n}$ will be more accurate if solar power samples data is available at a high resolution (e.g., 1 minute

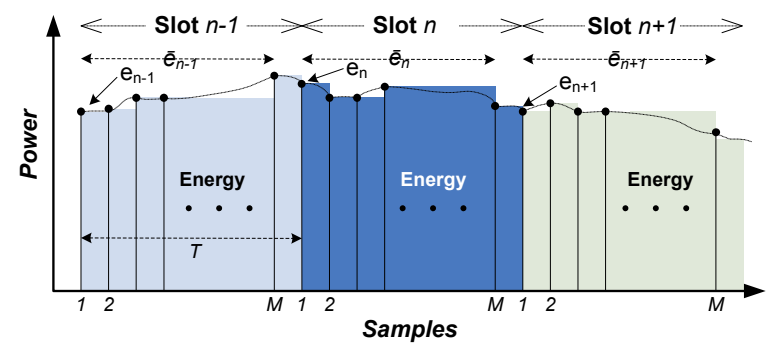

Fig. 4. A section of solar power samples profile showing slot boundaries, samples per slot, slot energy calculation, and mean slot power resolution compared to 5 minutes). This leads to realistic modeling of prediction error when using Equation 7.

Since determination of prediction accuracy needs to account for prediction error outcomes of a large number of sample points, a suitable average error function is required. Root Mean Squared Error (RMSE) is a commonly used measure of determining estimation accuracy [8], however, RMSE is sensitive to large outliers and its value is dependent on scale of data. This makes use of RMSE non-intuitive for evaluating harvested-energy prediction since sudden large fluctuations in solar energy profile are difficult to model with simplistic (heuristic) prediction algorithms and may give large error values (outliers) that can bias the average results. Mean Absolute Error (MAE) does not suffer from this aspect but it is also data scale dependent, making comparison of prediction performance across different solar power data sets non-intuitive. In this paper, we use Mean Absolute Percentage Error (MAPE) function (Equation 8) which is independent of data scale.

$$
M A P E=\frac{1}{T} \sum\left|\frac{\text { error }_{n}}{\bar{e}_{n}}\right|
$$

In Equation 8, $T$ is the the total number of predicted values. Similarly, we also define $M A P E^{\prime}$ based on error ${ }_{n}^{\prime}$ instead of error $_{n}$ (see Equation 6 and 7) and it will be used to compare prediction algorithm's parameters optimization results (Section IV-B). Since solar energy arrives in large bursts mainly during mid day, for harvested-energy management it is relevant to measure accuracy of prediction during this time. Therefore, night-time values (zero) where prediction is accurate but not useful, and small values at start/end of a day where prediction errors are not meaningful for evaluating prediction performance, should not be included in average prediction error calculation (Equation 8). This prevents the average prediction error to be influenced by points outside region of interest. The specific approach adopted to achieve this is mentioned in the next section.

\section{Evaluation AND Design EXPloration}

\section{A. Setup}

We evaluated the recently proposed prediction algorithm [5] using publicly available solar irradiance data of ten different sites [6], out of which six sites (Table I) are selected that demonstrate variety in solar energy profile variations. The use of multiple sites with large of number of recorded observations attempts to validate the proposed algorithm over long term deployment conditions (different number and distribution of sunny and cloudy days in each irradiance trace) and independent of the deployment location.

To present the algorithm evaluation results, the range of values used for the algorithm parameters are: $N=$ $\{288,96,72,48,24\}, 0 \leq \alpha \leq 1,2 \leq D \leq 20$ and $1 \leq K \leq 6$. These values are exhaustive to capture the main trends as shown by results in the following sections. For a given $N$, the objective is to find the optimized set of prediction algorithm parameters $\alpha$ (weighing factor), $K$ (previous slots) and $D$ (number of past days) for each solar 
TABLE I

DETAILS OF THE DATA SETS USED.

\begin{tabular}{ccccc}
\hline Data Set & Location & Observations & Days & Resolution \\
\hline SPMD & CO & 105,120 & 365 & 5 minutes \\
ECSU & NC & 105,120 & 365 & 5 minutes \\
ORNL & TN & 525,600 & 365 & 1 minute \\
HSU & CA & 525,600 & 365 & 1 minute \\
NPCS & NV & 525,600 & 365 & 1 minute \\
PFCI & AZ & 525,600 & 365 & 1 minute
\end{tabular}

power data set, which minimizes the average prediction error, $M A P E$. The sample values considered in the average error calculation are at least $10 \%$ of the peak value for reasons discussed in Section III. The evaluation is performed for days 21 to 365 as this allows matrix of past days samples $\mathbf{E}_{D \times N}$ used in the prediction algorithm to be filled for $D=20$, and it also ensures that an equal number and same sample values are used for average error calculation irrespective of number of past days $(D)$.

To evaluate the prediction algorithm on actual hardware, the following set-up has been used [9]:

- Test board: MSP-TS430PM64.

- Microcontroller: TI MSP430F1611 (3V@5MHz).

- Compiler: Code Composer Essentials 3.2.

Fig. 5 shows the steps in computation of prediction algorithm in hardware. Most of the time, micro-controller remains in deep sleep mode in which only the Wake-Up timer is running. The MSP430 wakes up according to the number of predictions per day $(N)$, enables the voltage reference used in Analog-to-Digital (A/D) conversion and waits in sleep mode until the voltage settles. It then launches the $\mathrm{A} / \mathrm{D}$ conversion and waits for it to complete (again in sleep mode). When A/D conversion is complete, it shuts down the voltage regulator, executes the prediction algorithm and re-enters in deep sleep mode.

\section{B. Prediction Algorithm Evaluation}

Having proposed the error evaluation function in Section III, we show the difference in results between average prediction error measurement using $M A P E$ (Equation 8), which uses average slot power to calculate error, compared to $M A P E^{\prime}$, which uses error between estimated and actual sampled power at the beginning of slot. Table II shows two sets of optimization results for $\alpha, D$ and $K$ with $N=48$ samples per day for different solar power data sets. In the first set, $M A P E^{\prime}$ has been used as the cost function while the second set has been obtained by minimizing $M A P E$ function. Note

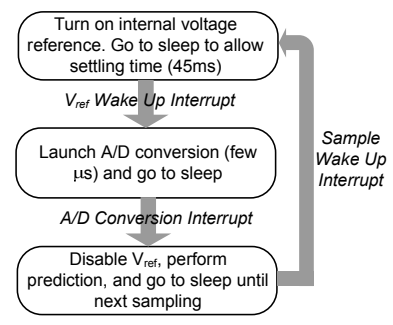

Fig. 5. Description of power value sampling and prediction sequence.
TABLE II

PREDICTION ERROR AND PARAMETER VALUES USING DIFFERENT ERROR EVALUATIONS AT $N=48$ FOR SIX SOLAR POWER DATA SETS.

\begin{tabular}{c|llll|llll}
\hline Data set & $\alpha$ & D & K & MAPE $^{\prime}$ & $\alpha$ & D & K & MAPE \\
\hline SPMD & 0.2 & 19 & 1 & $42.07 \%$ & 0.7 & 20 & 1 & $15.80 \%$ \\
ECSU & 0.2 & 20 & 2 & $32.89 \%$ & 0.7 & 20 & 3 & $13.45 \%$ \\
ORNL & 0.4 & 20 & 3 & $36.61 \%$ & 0.7 & 20 & 3 & $17.22 \%$ \\
HSU & 0.4 & 20 & 3 & $26.90 \%$ & 0.7 & 18 & 3 & $14.01 \%$ \\
NPCS & 0.0 & 15 & 1 & $17.17 \%$ & 0.6 & 20 & 2 & $8.06 \%$ \\
PFCI & 0.2 & 20 & 3 & $13.93 \%$ & 0.6 & 20 & 3 & $6.59 \%$ \\
\hline
\end{tabular}

that the values of average prediction errors for $M A P E$ are significantly lower compared to $M A P E^{\prime}$. Also, the obtained values of prediction algorithm parameters $(\alpha, D$, and $K)$ differ between the two error evaluations, especially the value of $\alpha$. These results indicate that prediction algorithm's parameters optimization is affected by choice of error function besides the difference in obtained average error values. As discussed in Section III, the average error values resulting from $M A P E$ optimization capture the differences in predicted and received energy more accurately.

Next, we evaluate the prediction algorithm using different values of prediction horizons. The two issues we want to address here are:

1) How much influence does varying prediction horizon or sampling rate per day $(N)$ have on prediction accuracy and associated overhead?

2) Based on analysis across multiple solar power data sets, can we arrive at some guidelines to simplify tuning of parameters $\alpha, K$ and $D$ independent of specific data set?

To address the first issue, Table III shows prediction error for five values of $N$ and the optimized values of parameters $K, \alpha$ and $D$ for each of six data sets. As can be seen for all data sets, prediction accuracy increases with increase in $N$, with predictions errors less than $9 \%$ in all cases at $N=288$, a gain of up to $9 \%$ compared to $N=48$.

Table IV gives the energy consumption of power sampling (A/D conversion) and prediction algorithm execution at some parameters' configurations. The energy consumption during system sleep mode per day is also given. As can be seen, the A/D conversion for sampling the power consumes the bulk of energy and prediction algorithm uses an additional $4 \mu \mathrm{J}$ to $9 \mu \mathrm{J}$ depending upon its parameters' values. Taking $5 \mu \mathrm{J}$ as roughly the typical energy consumption of prediction algorithm, the total energy consumption per day of prediction activity is given in last row of Table IV. If this is compared in context of energy consumption of sleep mode, it is interesting to note that the total energy consumption of the sampling and prediction activity combined for $N=48(2.880 \mathrm{~mJ}$ per day $)$ is still small compared to the total energy consumption of sleep mode (356mJ per day), indeed just $0.8 \%$. If we consider the extreme of $N=288(17.28 \mathrm{~mJ}$ per day), it is $4.85 \%$ of sleep mode energy consumption. Comparing the increase in overhead with increase in accuracy, it can be seen that using $N=288$ achieves an average error of less than $9 \%$, or an improvement of $7-10 \%$ in average error compared to $\mathrm{N}=48$ in high variability data sets (Table III). Fig. 6 gives the total energy consumption at different values of $N$ per day as a 
TABLE III

PREDICTION RESULTS AT DIFFERENT VALUES OF $N$.

\begin{tabular}{|c|c|c|c|c|c|c|}
\hline Data Set & $\mathbf{N}$ & $\alpha$ & D & $\mathbf{K}$ & MAPE & MAPE@K = 2 \\
\hline \multirow[t]{5}{*}{ SPMD } & 288 & 1 & $\mathrm{n} / \mathrm{a}$ & $\mathrm{n} / \mathrm{a}$ & $0 \dagger$ & $0 \dagger$ \\
\hline & 96 & 0.8 & 20 & 1 & $10.27 \%$ & $10.39 \%$ \\
\hline & 72 & 0.8 & 20 & 1 & $12.36 \%$ & $12.47 \%$ \\
\hline & 48 & 0.7 & 20 & 1 & $15.80 \%$ & $16.10 \%$ \\
\hline & 24 & 0.6 & 12 & 2 & $20.35 \%$ & $\mathrm{n} / \mathrm{a}$ \\
\hline \multirow[t]{5}{*}{ ECSU } & 288 & 1 & $\mathrm{n} / \mathrm{a}$ & $\mathrm{n} / \mathrm{a}$ & $0 \dagger$ & $0 \dagger$ \\
\hline & 96 & 0.8 & 20 & 2 & $9.39 \%$ & $\mathrm{n} / \mathrm{a}$ \\
\hline & 72 & 0.8 & 20 & 3 & $11.11 \%$ & $11.19 \%$ \\
\hline & 48 & 0.7 & 20 & 3 & $13.45 \%$ & $13.51 \%$ \\
\hline & 24 & 0.6 & 19 & 1 & $18.24 \%$ & $18.51 \%$ \\
\hline \multirow[t]{5}{*}{ ORNL } & 288 & 1 & $\mathrm{n} / \mathrm{a}$ & $\mathrm{n} / \mathrm{a}$ & $8.31 \%$ & $\mathrm{n} / \mathrm{a}$ \\
\hline & 96 & 0.8 & 20 & 3 & $14.42 \%$ & $14.47 \%$ \\
\hline & 72 & 0.8 & 20 & 4 & $15.72 \%$ & $15.88 \%$ \\
\hline & 48 & 0.7 & 20 & 3 & $17.22 \%$ & $17.43 \%$ \\
\hline & 24 & 0.6 & 12 & 2 & $21.43 \%$ & $\mathrm{n} / \mathrm{a}$ \\
\hline \multirow[t]{5}{*}{ HSU } & 288 & 0.9 & 20 & 1 & $6.00 \%$ & $6.01 \%$ \\
\hline & 96 & 0.8 & 20 & 4 & $10.80 \%$ & $10.88 \%$ \\
\hline & 72 & 0.8 & 20 & 5 & $12.11 \%$ & $12.30 \%$ \\
\hline & 48 & 0.7 & 18 & 3 & $14.01 \%$ & $14.11 \%$ \\
\hline & 24 & 0.7 & 12 & 2 & $19.19 \%$ & $\mathrm{n} / \mathrm{a}$ \\
\hline \multirow[t]{5}{*}{ NPCS } & 288 & 0.9 & 20 & 1 & $3.91 \%$ & $3.92 \%$ \\
\hline & 96 & 0.7 & 20 & 3 & $6.78 \%$ & $6.80 \%$ \\
\hline & 72 & 0.6 & 20 & 2 & $7.40 \%$ & $\mathrm{n} / \mathrm{a}$ \\
\hline & 48 & 0.6 & 20 & 2 & $8.06 \%$ & $\mathrm{n} / \mathrm{a}$ \\
\hline & 24 & 0.5 & 20 & 1 & $8.88 \%$ & $9.11 \%$ \\
\hline \multirow[t]{5}{*}{ PFCI } & 288 & 0.9 & 20 & 4 & $3.45 \%$ & $3.46 \%$ \\
\hline & 96 & 0.7 & 20 & 5 & $5.64 \%$ & $5.77 \%$ \\
\hline & 72 & 0.6 & 20 & 4 & $5.92 \%$ & $6.08 \%$ \\
\hline & 48 & 0.6 & 20 & 3 & $6.59 \%$ & $6.68 \%$ \\
\hline & 24 & 0.5 & 10 & 2 & $8.97 \%$ & $\mathrm{n} / \mathrm{a}$ \\
\hline
\end{tabular}

n/a: not applicable

$\dagger \mathrm{N}=288$ is not defined for this data set since the resolution of data set samples is 5 minutes

percentage of the sleep mode energy consumption.

Table III shows that as value of $N$ approaches 288, the value of $\alpha$ tends to 1 . Value of $\alpha \approx 1$ implies that prediction algorithm is mainly relying on the currently sampled power value to determine the predicted value, and $\alpha=1$ essentially means that current value can be used to predict the energy. These results show that using high values of $N$, need for using the prediction algorithm is reduced but at the same time energy consumption overhead is dominated by power sampling of ADC and not by prediction activity.

We now address the issue of simplifying tuning of prediction algorithm parameters across different solar power profiles to achieve low average errors in different working conditions:

- $D$ : Fig. 7 shows the values of $M A P E$ versus $D$ at $N=$ 48 using values of $\alpha$ and $K$ obtained in Table III. It can be seen that beyond a certain $D$ value, further gains in accuracy are small. $D$ can be set to value of $10-11$

TABLE IV

ENERGY CONSUMPTION OF POWER SAMPLING AND PREDICTION ALGORITHM.

\begin{tabular}{l|l}
\hline Hardware Activity & Energy/Cycle \\
\hline A/D conversion & $55 \mu \mathrm{J}$ \\
A/D conversion + Prediction $(\mathrm{K}=1, \alpha=0.7)$ & $58.6 \mu \mathrm{J}$ \\
A/D conversion + Prediction $(\mathrm{K}=7, \alpha=0.7)$ & $63.4 \mu \mathrm{J}$ \\
A/D conversion + Prediction $(\mathrm{K}=7, \alpha=0.0)$ & $61.5 \mu \mathrm{J}$ \\
Low power (sleep) mode 1.4 $\mu \mathrm{A} @ 3 \mathrm{~V}$ & $356 \mathrm{~mJ}$ per day \\
A/D conversion 48 samples per day@55 $\mu \mathrm{J}$ & $2640 \mu \mathrm{J}$ per day \\
A/D conversion + prediction 48 times per day@60 $\mu \mathrm{J}$ & $2880 \mu \mathrm{J}$ per day
\end{tabular}

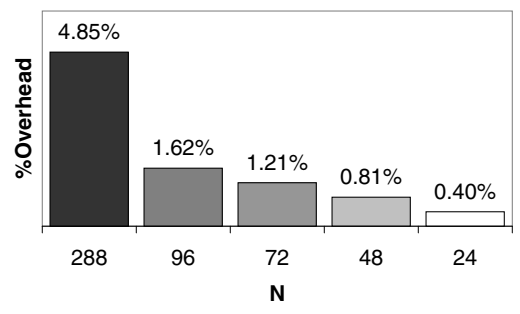

Fig. 6. Prediction algorithm overhead at different $N$

irrespective of the data set used to obtain low $M A P E$ while conserving samples storage memory requirement of prediction algorithm.

- $\alpha$ : Table III indicates that $\alpha=0.5$ to 0.6 gives minimum average error at $N=24$, and for $N=288 \alpha \approx 1$ is desirable. For other values of $N$ in between, $0.7 \leq \alpha \leq$ 0.8 with $48 \leq N \leq 96$ gives the minimum average error.

- $K$ : The last column of Table III show that $K=2$ gives an average error that is very close to minimum error value obtained for all data sets.

Based on empirical results, we have presented the above guidelines to achieve optimized design. Note that due to the heuristic nature of prediction algorithm and stochastic characteristic of solar energy profiles, values of $\alpha$ and $K$ need to be selected for different solar energy profiles. Consequently, we suggest guidelines instead of an exact method to determine pseudo-optimal values of $\alpha$ and $K$.

\section{Prediction with Dynamic Parameters Selection}

In Section IV-B we have shown that increasing the harvested-power sampling rate $(N)$ always results in higher prediction accuracy at a higher energy consumption overhead. We also showed that as more number of past days $(D)$ are considered, the average prediction error decreases noticeably initially, with the rate of decrease soon becoming insignificant. It can also be noted that across different data sets as well as at different values of $N$ for a given data set, the average prediction error was minimized for combination of parameters $\alpha$ and $K$ that varied for these different cases. From these observations, it can be concluded that although there is a fixed trend in average error values when $N$ or $D$ are varied, the average error value with a given $K$ and/or $\alpha$ depends on variations in solar power profile. In other words, for a

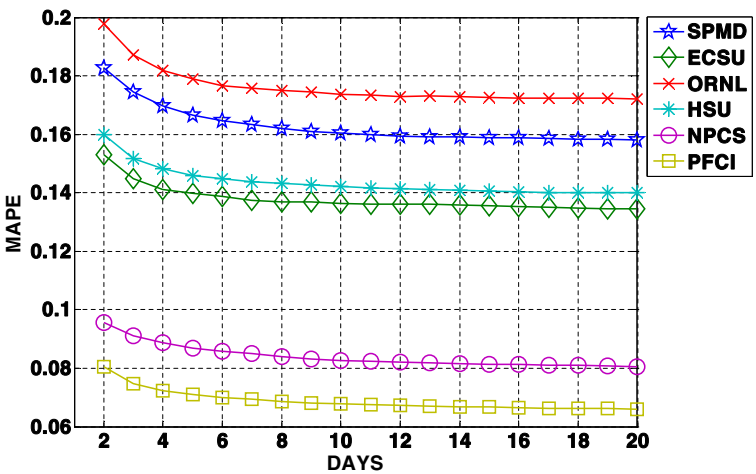

Fig. 7. $M A P E$ trends with increasing $D$ for different data sets 
given value of $N$ and $D$, values of $K$ and $\alpha$ may be varied at different points in a profile to minimize error at these points compared to using certain fixed values that minimize the average error across the whole profile (Section IV-B).

To demonstrate the potential gains in prediction accuracy by dynamically varying $\alpha$ and $K$, Table $\mathrm{V}$ gives the values of average errors with dynamically changing both $\alpha$ and $K$, changing only $K$ at a given $\alpha$ and vice versa. These averaged error $(M A P E)$ values in Table $\mathrm{V}$ are calculated using the minimum prediction error at every prediction, which is obtained by evaluating the Equation 1 for values of $0 \leq \alpha \leq 1$ and/or $1 \leq K \leq 6$ to obtain the minimum prediction error. This constitutes a clairvoyant approach to select the ideal values of $\alpha$ and/or $K$ that minimizes the prediction error. Table $\mathrm{V}$ also gives average error obtained using static parameters setting (from Table III) for comparison. When $K$ is dynamically changing, a fixed value of $\alpha$ has been chosen for which average error is minimum among other values of $\alpha$. The same consideration has been made when $\alpha$ is changed. Note that maximum gains in average error compared to static parameters algorithm are achieved when both $K$ and $\alpha$ are adapted, followed by adapting only $\alpha$ at a given $K$. Furthermore, these gains of dynamic algorithms compared to static algorithm increase as $N$ is decreased. This is a useful outcome since the implementation overhead of dynamic adjustment will be minimized at a smaller $N$. It is interesting to note that the dynamic algorithm accuracy at $N=48$ is higher than the accuracy of static algorithm at $N=288$. Observing the best values of $\alpha$ when $K$ is dynamically adjusted and vice versa (' $K$ only' and ' $\alpha$ only' columns), we find that these values of $\alpha$ and $K$ are different from the pseudo-optimum values suggested in Section IV-B. Lower values of $\alpha$ and higher $K$ values give better results when the other parameter ( $\alpha$ or $K)$ is dynamically set at every prediction. A low $\alpha$ implies that effect of conditioned average term (Equation 1 dominates while a high $K$ dampens the effect of conditioned average term due to consideration of more previous slots (Section II). It should be noted that the indicated error values with dynamic parameters' selection are minimum achievable since an ideal approach is used to select best parameters at every point. These results show that it is promising to develop dynamic parameters selection algorithms that can achieve less than $10 \%$ average error without the need to use higher sampling rates.

\section{CONCLUSION}

In this paper we have evaluated prediction accuracy of solar harvested-energy prediction based on recently reported prediction algorithm. To ensure that the obtained numbers were indicative of the real performance, we reconsidered the choice of error evaluation function to achieve this objective. Using data from multiple real solar power measurements and implementing prediction algorithm on actual hardware, different prediction horizons were tested and computation overhead was measured. Based on resulting values of algorithm parameters, we suggested guidelines to simplify parameters' tuning for different working conditions. Although this evaluation was
TABLE V

RESULTS FOR DYNAMIC PARAMETERS SELECTION VARYING BOTH $\alpha$ AND $K$, ONLY $K$ AT A FIXED $\alpha$ AND VICE VERSA.

\begin{tabular}{llllllll}
\hline \multirow{3}{*}{ Data Set } & & Static & K $+\alpha$ & \multicolumn{2}{c}{ K only } & \multicolumn{2}{c}{$\alpha$ only } \\
\hline SPMD & 288 & MAPE & MAPE & $\alpha$ & MAPE & K & MAPE \\
& 96 & $10.27 \%$ & $4.25 \%$ & 0.4 & $7.31 \%$ & 6 & $5.48 \%$ \\
& 72 & $12.36 \%$ & $5.13 \%$ & 0.3 & $8.54 \%$ & 6 & $6.47 \%$ \\
& 48 & $15.80 \%$ & $6.43 \%$ & 0.3 & $10.63 \%$ & 6 & $8.21 \%$ \\
& 24 & $20.35 \%$ & $6.95 \%$ & 0.3 & $13.08 \%$ & 3 & $11.21 \%$ \\
\hline ECSU & 288 & $0.00 \%$ & $0.00 \%$ & 1 & $0.00 \%$ & n/a & 0 \\
& 96 & $9.39 \%$ & $3.76 \%$ & 0.3 & $6.32 \%$ & 6 & $4.85 \%$ \\
& 72 & $11.11 \%$ & $4.44 \%$ & 0.3 & $7.40 \%$ & 6 & $5.68 \%$ \\
& 48 & $13.45 \%$ & $5.37 \%$ & 0.3 & $8.92 \%$ & 6 & $6.93 \%$ \\
& 24 & $18.24 \%$ & $6.16 \%$ & 0.3 & $11.25 \%$ & 3 & $10.37 \%$ \\
\hline ORNL & 288 & $8.31 \%$ & $3.85 \%$ & 0.2 & $6.07 \%$ & 6 & $4.68 \%$ \\
& 96 & $14.42 \%$ & $6.40 \%$ & 0 & $9.35 \%$ & 6 & $7.69 \%$ \\
& 72 & $15.72 \%$ & $6.72 \%$ & 0 & $10.09 \%$ & 6 & $8.10 \%$ \\
& 48 & $17.22 \%$ & $7.38 \%$ & 0.1 & $11.34 \%$ & 6 & $9.26 \%$ \\
& 24 & $21.43 \%$ & $7.30 \%$ & 0.2 & $12.94 \%$ & 3 & $12.03 \%$ \\
\hline HSU & 288 & $6.00 \%$ & $2.75 \%$ & 0.3 & $4.46 \%$ & 6 & $3.43 \%$ \\
& 96 & $10.80 \%$ & $4.60 \%$ & 0.1 & $7.19 \%$ & 6 & $5.76 \%$ \\
& 72 & $12.11 \%$ & $5.15 \%$ & 0.2 & $8.14 \%$ & 6 & $6.49 \%$ \\
& 48 & $14.01 \%$ & $5.52 \%$ & 0.2 & $9.32 \%$ & 6 & $7.36 \%$ \\
& 24 & $19.19 \%$ & $5.92 \%$ & 0.3 & $11.21 \%$ & 3 & $10.11 \%$ \\
\hline
\end{tabular}

done using a recently proposed prediction algorithm, it is also applicable to other solar energy prediction algorithms since the dimensions or parameters considered in this paper are general in context of prediction algorithms. Finally, we explored the gains of extending to dynamic parameters selection algorithm and found that more than $10 \%$ gains can be achieved depending upon the length of prediction horizon.

\section{ACKNOWLEDGMENT}

The first two authors would like to thank the EPSRC-UK for funding this work in part under grant number EP/E035965/1. Also, this work is partially supported by the Spanish Government Research Grants TIN2008-00508 and CSD00C-0720811.

\section{REFERENCES}

[1] W. K. Seah, Z. A. Eu, and H.-P. Tan, "Wireless sensor networks powered by ambient energy harvesting (WSN-HEAP) - survey and challenges," in Proc. VITAE '09, May 2009, pp. 1-5.

[2] A. Kansal, J. Hsu, S. Zahedi, and M. B. Srivastava, "Power management in energy harvesting sensor networks," ACM TECS, vol. 6, no. 4, p. 32, 2007.

[3] C. Moser, L. Thiele, D. Brunelli, and L. Benini, "Robust and low complexity rate control for solar powered sensors,' in Proc. DATE '08, March 2008, pp. 230-235.

[4] D. K. Noh, L. Wang, Y. Yang, H. K. Le, and T. Abdelzaher, "Minimum variance energy allocation for a solar-powered sensor system," in DCOSS '09: Proceedings of the 5th IEEE International Conference on Distributed Computing in Sensor Systems. Springer-Verlag, 2009, pp. 44-57.

[5] J. Recas, C. Bergonzini, D. Atienza, and T. S. Rosing, "Prediction and management in energy harvested wireless sensor nodes," in Proc. VITAE '09, May 2009, pp. 6-10.

[6] "NREL: Measurement and Instrumentation Data Center (MIDC) Home Page." [Online]. Available: http://www.nrel.gov/midc/

[7] C. Bergonzini, D. Brunelli, and L. Benini, "Algorithms for harvested energy prediction in batteryless wireless sensor networks," in Advances in sensors and Interfaces, 2009. IWASI 2009. 3rd International Workshop on, June 2009, pp. 144-149.

[8] R. J. Hyndman and A. B. Koehler, "Another look at measures of forecast accuracy," International Journal of Forecasting, vol. 22, no. 4, pp. $679-$ 688, 2006.

[9] “Texas instruments." [Online]. Available: http://www.ti.com/ 